\title{
Correlações genéticas e fenotípicas entre características de tipo e produção de leite em bovinos da raça Holandesa
}

[Genetic and phenotypic correlations between type traits and milk production in Holstein cattle]

\author{
A.M.C. Esteves ${ }^{1}$, J.A.G. Bergmann ${ }^{1}$, M.C. Durães ${ }^{2}$, C.N. Costa ${ }^{2}$, H.M. Silva ${ }^{1}$ \\ ${ }^{1}$ Escola de Veterinária da UFMG \\ Caixa Postal 567 \\ 30123-970 - Belo Horizonte, MG \\ ${ }^{2}$ EMBRAPA - Gado de Leite - Juiz de Fora, MG
}

\begin{abstract}
RESUMO
Correlações genéticas e fenotípicas entre 21 características lineares de tipo e produção de leite em até 305 dias de lactação foram estimadas pela máxima verossimilhança restrita sob modelo animal, a partir de dados da Associação de Criadores de Gado Holandês de Minas Gerais. Nas análises das características lineares, os efeitos fixos utilizados no modelo incluíram estádio da lactação, idade do animal, grupo contemporâneo de rebanho, ano e estação de classificação, e o efeito aleatório do pai da vaca. As características lineares que apresentaram maiores correlações genéticas com produção de leite foram largura do úbere posterior $(0,60)$ e largura de garupa $(0,37)$, indicando não haver antagonismo genético entre a seleção para essas características e a produção de leite. As correlações fenotípicas entre características lineares de tipo e produção de leite foram, em geral, menores do que as correlações genéticas correspondentes. As correlações fenotípicas entre as características lineares apresentaram-se altas dentro das seções de conformação/capacidade e de sistema mamário. Correlações genéticas entre algumas características lineares foram altas, possibilitando a exclusão de algumas delas do programa de classificação linear adotado para a raça Holandesa no Brasil.
\end{abstract}

Palavras-chave: bovino, característica linear, correlação entre tipo e produção

\begin{abstract}
Genetic and phenotypic correlations between 21 linear type traits and 305-day milk production were estimated by restrict maximum likelihood methodology, in a multiple-trait animal model, using data from Associação de Criadores de Gado Holandês de Minas Gerais. The highest genetic correlations between milk production and rear udder width (.60), and rump width (.37), indicated no genetic antagonism between selection for these type traits and gains for milk production. In general, phenotypic correlations were lower than genetic correlations between milk production and linear type traits. Phenotypic correlations between linear traits were greater for conformation traits and udder traits. Some type traits presented high genetic correlations between them, suggesting that some of them could be excluded from the genetic programs for linear type traits adopted by Holstein Breed Association in Brazil.
\end{abstract}

Keywords: cattle, Holstein, linear type trait, genetic and phenotypic correlations

Recebido para publicação em 16 de outubro de 2003

Recebido para publicação, após modificações, em 23 de março de 2004

E-mail: alessandro@genespecuaria.com.br 


\section{Esteves et al.}

\section{INTRODUÇÃO}

Ênfase no processo de seleção de gado leiteiro é dada para características de produção porque vacas de alta produção geram mais receita. Em geral, a receita será ainda maior se a vaca tiver alta produção de leite nas primeiras lactações e se apresentar funcional por período extenso dentro do rebanho. Porém, a seleção praticada somente para produção de leite pode diminuir o mérito de outras características (Misztal et al., 1992).

Como a produção de leite é a característica mais importante em programas de melhoramento de gado leiteiro, faz-se necessário avaliar a sua associação com outras características, bem como analisar como essas se comportam quando a seleção é feita para produção de leite. Características de tipo devem merecer atenção quando o objetivo é maximizar a vida produtiva do animal, evitando descartes precoces por problemas de aprumos e ligamentos, dentre outros. Grande parte da literatura a respeito desse assunto, quase toda em países de clima temperado, conclui que a seleção fenotípica com base em escores lineares de conformação tem valor limitado, em conseqüência da baixa correlação genética entre caracteres morfológicos e produtivos, da grande influência do meio ambiente e da ação genética não aditiva.

São escassos os trabalhos de pesquisa sobre características lineares de tipo no Brasil. Diferente do que ocorre nos países de primeiro mundo, no Brasil não existe teste de progênie para características lineares. Menor importância, então, é dada para os resultados de escores lineares dos animais, restando aos técnicos e produtores a necessidade de conhecer as relações existentes entre determinadas características lineares e como a seleção para tipo poderia refletir na produção de leite.

Este trabalho teve por objetivos estimar as correlações genéticas e fenotípicas entre as características lineares e produção de leite em até 305 dias de lactação e correlações genéticas e fenotípicas entre características lineares de tipo.

\section{MATERIAL E MÉTODOS}

Os dados de classificação linear utilizados neste estudo foram coletados por técnicos da Associação de Criadores de Gado Holandês de Minas Gerais (ACGHMG), de 1995 a 1998. O arquivo de classificação linear apresentou 2.122 observações, incluindo 21 características lineares avaliadas, com resultados de escores que variaram de 1 a 9 pontos, mais a pontuação final, que variou de 55 a 90 pontos (Esteves et al., 2004).

Os dados de produção de leite em até 305 dias de lactação também foram provenientes dos arquivos da ACGHMG e referem-se às lactações encerradas no período acima citado, gerando um total de 1.975 observações.

O método utilizado nas análises iniciais foi o dos quadrados mínimos, com os componentes de variância estimados pelo método III de Henderson (1953). Para avaliar o efeito dos diversos fatores de ambiente sobre as características lineares de tipo, os efeitos de rebanho, ano e estação da classificação foram combinados em único efeito fixo denominado rebanho-ano-estação da classificação (RAEC). Além da variável RAEC, o modelo utilizado para as características lineares também incluiu os efeitos fixos de estádio da lactação e idade do animal, e o efeito aleatório de reprodutor pai da vaca. A descrição completa da modelagem e da avaliação dos efeitos fixos sobre as características de tipo pode ser encontrada em Esteves et al. (2004).

Para a característica produção de leite em até 305 dias foram agrupados os efeitos de rebanho, ano e estação da parição em um único efeito fixo denominado rebanho-ano-estação da parição (RAEP). O modelo utilizado nas análises iniciais incluiu os efeitos fixos de RAEP e idade do animal, além do efeito aleatório de reprodutor pai da vaca. Para esta característica produtiva, o modelo matemático utilizado foi o seguinte

$\mathrm{Y}_{\mathrm{ijklm}}=\mu+\mathrm{RAEP}_{\mathrm{i}}+\mathrm{A}_{\mathrm{j}}+\mathrm{S}_{1}+\mathrm{e}_{\mathrm{ijlm}}$, em que:

$\mathrm{Y}_{\mathrm{ijklm}}=$ observação da produção de leite em até 305 dias do indivíduo m;

$\mu=$ média da população;

RAEP $_{\mathrm{i}}=$ efeito fixo da subclasse $\mathrm{i}$ para rebanho, ano e estação de parição;

$\mathrm{A}_{\mathrm{j}}=$ efeito fixo da subclasse $\mathrm{j}$ para idade da vaca ao parto;

$\mathrm{S}_{1}=$ efeito aleatório do pai da vaca;

$\mathrm{e}_{\mathrm{m}}=$ efeito aleatório do erro associado à cada 
observação.

Os modelos de análise conjunta de duas características foram utilizados para determinação das correlações genéticas e fenotípicas entre as características de tipo e a produção de leite em até 305 dias, usando o modelo animal. As análises foram realizadas utilizando-se o aplicativo multiple trait derivative free restrict maximum likelihood (MTDFREML), descrito por Boldman et al. (1995). Nas análises conjuntas de duas características os valores iniciais adotados foram os encontrados a partir dos componentes de variância obtidos nas análises pelo método REML, com uma característica.

Os valores iniciais das covariâncias genética e residual foram obtidos pela fórmula de determinação das correlações conforme descrito por Henderson e Quaas (1976). Usando estimativas de variância obtidas neste estudo e valores de correlações encontrados na literatura determinaram-se os valores de covariância entre as características mediante aplicação da fórmula

$\sigma_{\mathrm{AB}}=\mathrm{r}_{\mathrm{AB}} \sqrt{\sigma_{\mathrm{A}}^{2}} \cdot \sigma_{\mathrm{B}}^{2}$, em que

$\sigma_{\mathrm{AB}}=$ covariância entre a característica A e B;

$\mathrm{r}_{\mathrm{AB}}=$ correlação entre a característica A e B;

$\sigma_{\mathrm{A}}^{2}=$ variância da característica $\mathrm{A}$;

$\sigma^{2}{ }_{B}=$ variância da característica $B$.

\section{RESULTADOS E DISCUSSÃO}

As estimativas de correlações fenotípicas entre os escores lineares foram, quase sempre, menores que as estimativas de correlações genéticas correspondentes (Tab. 1). Estes resultados estão de acordo com vários autores (Klassen et al., 1992; Mrode e Swanson, 1994; Brotherstone, 1994).

Nenhuma associação direta foi verificada entre as características de úbere $\mathrm{e}$ as de conformação/capacidade. O mesmo ocorreu para as características de garupa e de sistema mamário, com exceção da largura da garupa e largura do úbere, que apresentaram correlação fenotípica moderada $(0,30)$, sugerindo que vacas com garupas amplas tenderam a apresentar úbere posterior mais largo. Isto, também, foi verificado por outros autores (Lawstuen et al., 1987; 0,24; Mrode e Swanson, 1994; 0,42).
Muitas correlações genéticas positivas e elevadas foram verificadas entre as características de conformação/capacidade (Tab. 1). A alta correlação observada entre tamanho e estatura $(0,82)$ evidencia associação genética entre elas e indica que uma delas poderia ser removida do sistema de classificação linear atualmente adotado pela raça Holandesa no Brasil. Altas correlações entre as duas características foram também encontradas por Schaeffer $(1983 ; 0,96)$ e Klassen et al. (1992; 0,97).

As correlações genéticas entre algumas características do sistema mamário foram elevadas. Comprimento das tetas apresentou correlação alta e negativa com outras características, como largura do úbere posterior, colocação das tetas anteriores e ligamento suspensório mediano $(-0,65 ;-0,54$ e $-0,51$, respectivamente), indicando que a seleção para tetos mais longos poderá promover redução da qualidade das outras características do sistema mamário. Resultados semelhantes foram citados por Short et al. (1991), porém em menor magnitude. A colocação das tetas anteriores e tetas posteriores apresentou correlação genética alta e positiva $(0,71)$. Assim, poder-se-ia conjecturar ser necessária a avaliação de apenas uma dessas características. Esta possibilidade é reforçada pela alta correlação entre as duas características também encontradas por Klassen et al. (1992; 0,85). A largura e a altura do úbere posterior apresentaram-se positiva e moderadamente correlacionadas $(0,42)$, sendo este resultado importante, uma vez que busca-se como ideal da vaca leiteira a apresentação de úberes posteriores largos e de inserção alta.

Correlações genéticas negativas foram verificadas, principalmente, entre ângulo do casco e outras características lineares. Maior expressão pode ser observada nas suas relações com ligamento e altura do úbere (-0,44 e -0,29, respectivamente). Vista lateral das pernas, outra característica da seção pernas/pés, também apresentou correlações negativas com algumas características do sistema mamário. Esta relação genética adversa, presente entre as seções de pernas/pés e de úbere, é preocupante, uma vez que em muitos programas de melhoramento propostos, estas características estão incluídas nos índices de seleção, juntamente com as características de produção. 


\section{Esteves et al.}

Tabela 1. Correlações genéticas (acima da diagonal) e fenotípicas (abaixo da diagonal) entre as características lineares de tipo

EST NCO TAM LTO PCO FLO NGA LGA ACA QOS PPE INS CPT ALT LPO CLA CLP PUB TEX LIG ANG PFI

$\begin{array}{llllllllllllllllllllllll}\text { EST }^{1} & 0,66 & 0,82 & 0,52 & 0,47 & 0,54 & 0,54 & 0,48 & 0,48 & 0,18 & -0,35 & -0,10 & -0,13 & 0,22 & 0,54 & 0,60 & 0,19 & 0,58 & 0,52 & 0,24 & 0,70 & 0,81\end{array}$

$\begin{array}{llllllllllllllllllllll}\text { NCO } & 0,28 & 0,59 & 0,37 & 0,60 & 0,37 & -0,10 & 0,27 & 0,38 & 0,07 & 0,20 & 0,16 & -0,37 & 0,17 & 0,18 & 0,09 & 0,23 & -0,17 & 0,32 & -0,02 & 0,73 & 0,70\end{array}$

$\begin{array}{llllllllllllllllllllll}\text { TAM } & 0,71 & 0,31 & 0,81 & 0,69 & 0,56 & 0,50 & 0,17 & 0,52 & -0,25 & -0,45 & 0,00 & 0,02 & -0,15 & 0,35 & 0,43 & 0,11 & 0,48 & 0,10 & 0,02 & 0,44 & 0,66\end{array}$

LTO $\quad 0,36 \quad 0,26 \quad 0,60 \quad \begin{array}{llllllllllllllllll}0,64 & -0,14 & -0,02 & 0,29 & 0,10 & 0,21 & 0,25 & 0,14 & 0,09 & 0,23 & 0,46 & -0,03 & 0,13 & 0,00 & 0,13 & -0,21 & 0,26 & 0,51\end{array}$

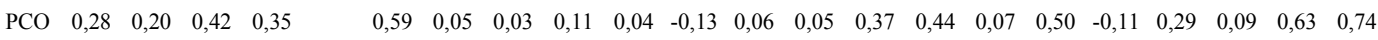

FLO $\quad 0,21 \quad 0,12 \quad 0,11 \quad-0,03 \quad 0,20 \quad \begin{array}{llllllllllllllll}0,16 & 0,12 & 0,18 & 0,28 & -0,05 & 0,31 & -0,25 & 0,51 & 0,39 & 0,17 & 0,09 & 0,02 & 0,41 & -0,08 & 0,58 & 0,57\end{array}$

NGA $\quad 0,05 \quad-0,11 \quad-0,02 \quad-0,08 \quad-0,07 \quad 0,12$

$\begin{array}{lllllllllllllll}0,19 & -0,20 & 0,12 & -0,38 & 0,09 & -0,01 & 0,00 & 0,43 & 0,24 & -0,26 & 0,21 & 0,02 & 0,18 & -0,04 & 0,12\end{array}$

LGA $\quad 0,32 \quad 0,12 \quad 0,21 \quad 0,12 \quad 0,13 \quad 0,16 \quad 0,01 \quad \begin{array}{llllllllllllll}0,0,02 & 0,02 & 0,46 & 0,10 & -0,14 & 0,05 & 0,73 & 0,22 & -0,10 & 0,35 & 0,25 & 0,06 & 0,36 & 0,40\end{array}$

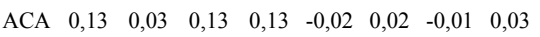

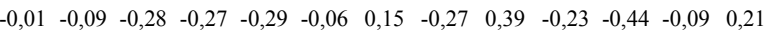

QOS $\quad 0,01 \quad 0,05 \quad-0,13 \quad-0,22 \quad 0,02 \quad 0,19 \quad-0,06 \quad 0,06 \quad-0,01$

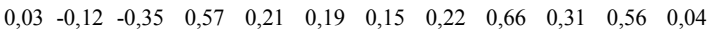

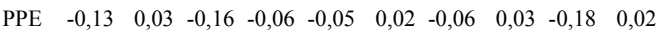

$\begin{array}{lllllllllll}0,15 & -0,18 & 0,38 & 0,31 & -0,07 & -0,21 & -0,33 & -0,07 & -0,09 & 0,26 & -0,06\end{array}$

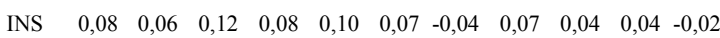

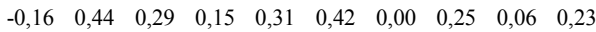

$\begin{array}{lllllllllllll}\text { CPT } & 0,03 & -0,03 & 0,06 & 0,04 & 0,04 & 0,00 & -0,01 & 0,00 & -0,02 & -0,05 & -0,07 & -0,04\end{array}$

$\begin{array}{llllllllll}-0,27 & -0,65 & -0,54 & 0,02 & -0,32 & -0,52 & -0,51 & -0,43 & -0,51\end{array}$

$\begin{array}{llllllllllllll}\text { ALT } & 0,14 & 0,07 & 0,06 & 0,03 & 0,11 & 0,16 & -0,06 & 0,19 & -0,00 & 0,17 & -0,05 & 0,17 & 0,02\end{array}$

$\begin{array}{llllllll}0,58 & 0,00 & 0,68 & 0,09 & 0,33 & 0,23 & 0,70 & 0,52\end{array}$

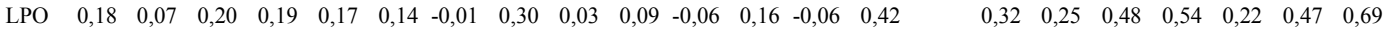

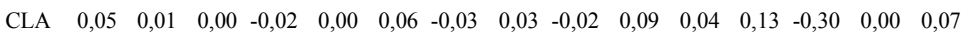

$\begin{array}{llllll}0,71 & 0,20 & 0,28 & 0,52 & 0,12 & 0,34\end{array}$

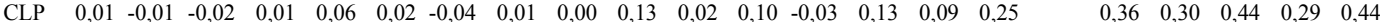

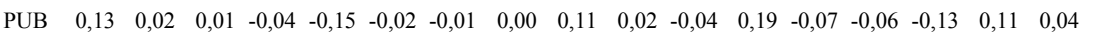

$\begin{array}{llll}0,14 & 0,33 & 0,37 & 0,02\end{array}$

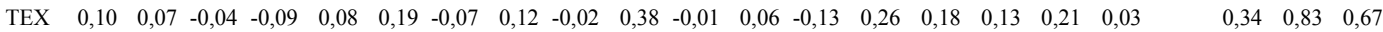

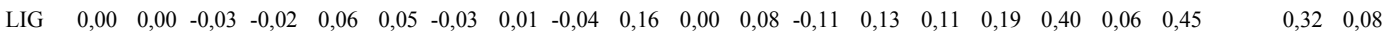

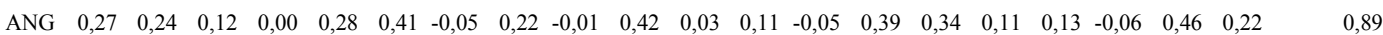

$\begin{array}{lllllllllllllllllllllll}\text { PFI } & 0,44 & 0,22 & 0,38 & 0,23 & 0,35 & 0,29 & -0,09 & 0,29 & 0,12 & 0,23 & -0,14 & 0,35 & -0,12 & 0,40 & 0,44 & 0,17 & 0,19 & 0,05 & 0,41 & 0,26 & 0,54\end{array}$

${ }^{1} \mathrm{EST}=$ estatura; NCO = nivelamento da linha superior; TAM = tamanho; LTO = largura torácica; PCO = profundidade corporal; $\mathrm{FLO}$ = força lombar; NGA = nivelamento da garupa; LGA = largura da garupa; ACA = ângulo do casco; QOS = qualidade óssea; $\mathrm{PPE}=$ vista lateral das pernas; INS = inserção do úbere anterior; CPT = comprimento das tetas; ALT = altura do úbere posterior; LPO = largura do úbere posterior; CLA = colocação das tetas anteriores; CLP = colocação das tetas posteriores; PUB = profundidade do úbere; TEX = textura do úbere; $\mathrm{LIG}=$ ligamento suspensório mediano do úbere; $\mathrm{ANG}=$ angulosidade; $\mathrm{PFI}=$ pontuação final

Correlações altas e positivas foram observadas entre pontuação final e diversas características lineares. Sendo a pontuação uma característica que abrange todos outros escores, suas relações genéticas merecem atenção, principalmente quando se estuda a formação de índices de seleção para uma raça ou rebanho leiteiro. Selecionando-se para altas pontuações finais, esperar-se-ia resposta equilibrada entre as diversas características, resultando em animais mais altos, nivelados na linha superior, pesados, profundos, angulosos, de lombos fortes, com úberes posteriores altos, largos e de boa textura.
Em relação aos diversos trabalhos publicados, pode-se verificar que as correlações genéticas estimadas neste estudo foram, em geral, ligeiramente superiores. Isto foi verificado mais acentuadamente quando estes resultados foram comparados com outros trabalhos que utilizaram modelo touro (Meyer et al., 1987; Short et al., 1991). De forma similar, Ducrocq (1993) encontrou correlações genéticas entre as características de úbere mais altas do que as verificadas em média na literatura em trabalhos que não utilizaram modelo animal em avaliações REML. Deve-se salientar que estes resultados 
poderiam também ter sido influenciados pelo relativamente pequeno número de observações de escores lineares considerados neste trabalho e pela utilização de resultados de classificações de todas as ordens de lactação.

Em geral, as correlações fenotípicas observadas entre as características lineares e a produção de leite em até 305 dias foram baixas, próximas de zero, como podem ser observadas na Tab. 2. Similarmente, vários autores observaram baixas correlações fenotípicas entre características lineares e produção de leite (Meyer et al., 1987; Mrode e Swanson, 1994; Brotherstone, 1994).

Tabela 2. Correlações genéticas e fenotípicas entre as características lineares de tipo e produção de leite

\begin{tabular}{lcc} 
Característica linear & $\begin{array}{c}\text { Correlação } \\
\text { fenotípica }\end{array}$ & $\begin{array}{c}\text { Correlação } \\
\text { genética }\end{array}$ \\
\hline Estatura & 0,06 & 0,13 \\
Nivelamento da linha superior & $-0,03$ & $-0,25$ \\
Tamanho & 0.01 & $-0,06$ \\
Largura torácica & 0,02 & 0,07 \\
Profundidade corporal & 0,04 & $-0,02$ \\
Força lombar & 0,11 & 0,05 \\
Nivelamento da garupa & 0,03 & 0,07 \\
Largura da garupa & 0,09 & 0,37 \\
Ângulo do casco & 0,01 & 0,30 \\
Qualidade óssea & 0,05 & 0,04 \\
Vista lateral das pernas & $-0,02$ & 0,22 \\
Inserção do úbere anterior & $-0,07$ & $-0,31$ \\
Comprimento das tetas & $-0,04$ & $-0,10$ \\
Altura do úbere posterior & 0,12 & 0,30 \\
Largura do úbere posterior & 0,16 & 0,60 \\
Colocação das tetas anteriores & $-0,02$ & $-0,09$ \\
Colocação das tetas posteriores & 0,01 & 0,09 \\
Profundidade de úbere & $-0,10$ & $-0,15$ \\
Textura de úbere & 0,13 & 0,35 \\
Ligamento suspensório mediano & 0,05 & $-0,04$ \\
Angulosidade & 0,14 & 0,19 \\
Pontuação final & 0,15 & 0,11 \\
\hline
\end{tabular}

As correlações fenotípicas entre características lineares de tipo e produção de leite foram moderadas, variando de $-0,10$ até 0,16 . Os maiores valores foram observados entre produção de leite e largura do úbere posterior, pontuação final e angulosidade $(0,16 ; 0,15$ e 0,14, respectivamente). Mrode e Swanson (1994) também verificaram correlações moderadas e positivas entre produção e as características largura do úbere posterior e angulosidade $(0,25 \mathrm{e}$ 0,20 respectivamente).
Em estudo realizado com dados brasileiros, Mcmanus e Saueressig (1997) também encontraram baixas correlações fenotípicas entre produção de leite e características lineares. Os autores observaram correlações fenotípicas moderadas e negativas entre produção de leite e as características de conformação/capacidade, indicando que animais com aparência mais forte produziram mais leite. A largura posterior do úbere teve correlação média e positiva com produção, mostrando que vacas com úberes mais largos produziram mais leite.

Correlações fenotípicas desfavoráveis foram observadas entre produção de leite em até 305 dias e as características profundidade do úbere e inserção do úbere anterior (- 0,10 e $-0,07$, respectivamente). De maneira mais expressiva, estas estimativas foram confirmadas por Brotherstone (1994), que encontrou -0,28 e -0,20 de correlação entre produção e profundidade do úbere e entre produção e inserção do úbere, respectivamente.

Algumas características, como peso corporal, não têm tido seu comportamento claramente definido, levando a resultados variados de correlação com produção de leite. Esperar-se-ia que vacas mais pesadas teriam maior capacidade de produção (Bowden, 1982). Persaud et al. (1991) concluíram que peso corporal teve correlação negativa com eficiência, influenciando a economicidade da atividade leiteira. Isto faz do peso corporal uma característica problemática de ser considerada em programas de melhoramento genético de rebanhos leiteiros (Hietanen e Ojala, 1995). A baixa correlação fenotípica entre produção de leite e tamanho do animal $(0,01)$ não sugere que vacas mais pesadas sejam indicativas das mais produtivas.

A maior correlação genética entre produção e tipo foi observada para largura do úbere posterior, 0,60. A seleção para animais com úberes largos é desejável e poderá levar a aumento da produção de leite. Juntamente com a largura, a altura do úbere posterior também apresentou correlação moderada e positiva com produção $(0,30)$. Sendo estas correlações favoráveis, ambas as características poderiam ser utilizadas na seleção por produtores que visam altas produções nas lactações, mas que não abrem mão de possuírem animais com boa 


\section{Esteves et al.}

conformação.

Outras características do sistema mamário, como profundidade do úbere e inserção do úbere anterior, apresentaram correlações desfavoráveis com produção (-0,15 e -0,31, respectivamente). Aparentemente, vacas de maior produção tenderam a apresentar úberes mais profundos e fracamente inseridos. Portanto, esta relação desfavorável deve ser observada com atenção quando a seleção para leite é aplicada sem restrições para tipo, uma vez que a conformação do úbere é, também, importante para a funcionalidade do animal. Da mesma maneira, Norman e Van Vleck (1972) sugeriram que úberes mais profundos seriam bons preditores de maior produção de leite, a partir da correlação genética de $-0,27$ com produção. Porém, como comentado por Young et al. (1960), é questionável se úberes profundos e mal inseridos seriam mais desejáveis, uma vez que existem evidências de sua associação com o aumento da incidência de mastites. Zwaar (1999) salienta também que a profundidade do úbere é a característica que resulta em maior taxa de descarte em relação às outras, ou seja, úberes mais profundos são os maiores responsáveis pela exclusão de animais dos rebanhos.

Além dessas características, textura do úbere apresentou-se como boa preditora de produção de leite, com correlação genética de 0,35. Ademais, sua alta correlação com outras características lineares do sistema mamário favorece, também, sua utilização na seleção para animais mais produtivos.

A segunda maior correlação observada entre produção de leite e tipo foi para largura da garupa (0,37). Assim, vacas com garupas amplas têm potencial para maior produção de leite.

A correlação entre produção de leite e ângulo do casco $(0,30)$ foi superior à verificada, em média, na literatura. Autores como Short e Lawlor (1992) e Brotherstone (1994) encontraram correlações muito baixas entre produção e ângulo do casco, 0,02 e $-0,02$, respectivamente. Os resultados do presente estudo indicam, entretanto, que para as condições do país, o ângulo do casco poderia ter importância como componente da produção de leite. Se considerada nos programas de seleção, dever-se-ia buscar animais com talões menos inclinados, que caracterizam boa qualidade dos cascos, necessárias às condições de ambiente e manejo prevalentes das fazendas leiteiras. Junto com ângulo do casco, a vista lateral das pernas também apresentou correlação mais alta $(0,22)$ do que aquelas verificadas em outros trabalhos, reforçando a importância da seção pernas/pés para os rebanhos de vacas Holandesas de Minas Gerais. Estudos têm comprovado a grande influência dos compostos de pernas/pés e de úbere na vida útil produtiva da vaca leiteira (Short e Lawlor, 1992). Principalmente em sistemas de alta produção, faz-se necessário que $o$ animal seja funcional e que não apresente alterações locomotoras e mamárias.

Outras correlações genéticas verificadas entre características lineares e produção de leite em até 305 dias foram de menor valor e, portanto, menos importantes para serem consideradas em programas de seleção para produção leiteira.

\section{CONCLUSÕES}

As correlações genéticas observadas entre produção de leite e as diversas características lineares revelaram possíveis ganhos para produção quando a seleção para algumas características de tipo é efetuada, podendo ser consideradas na formação de índices de seleção para determinadas raças ou rebanhos leiteiros, que visem atender ao mercado específico, seja para alta produção de leite ou seja para venda de animais de melhor constituição morfológica. As altas correlações genéticas entre algumas características lineares, associadas com a necessidade de maior objetividade na prática da classificação feita no campo, fornecem subsídios para reestruturação do sistema de classificação adotado para a raça Holandesa no Brasil. Poderse-ia recomendar a eliminação de algumas características lineares, tendo em vista a alta correlação genética encontrada entre elas.

\section{REFERÊNCIAS BIBLIOGRÁFICAS}

BOLDMAN, K.G.; KRIESE, L.A.; VAN VLECK, L.D. et al. A manual for use of MTDFREML-a set of programs to obtain estimates of variance and covariance. Lincoln: USDA-ARS, 1995. 120p. 
BOWDEN, V. Type classification in dairy cattle: a review. Anim. Breed. Abstr., v.50, p.147-161, 1982.

BROTHERSTONE, S. Genetic and phenotypic correlations between linear type traits and production traits in Holstein-Friesian dairy cattle. Anim. Prod., v.59, p.183-187, 1994.

DUCROCQ, V. Genetic parameters for type traits in the French Holstein breed based on a multiple-trait animal model. Livest. Prod. Sci., v.36, p.143-156, 1993.

ESTEVES, A.M.C.; BERGMANN, J.A.G.; DURÃES, M.C. et al. Estudo dos efeitos de ambiente sobre as características lineares de tipo em rebanhos bovinos da raça Holandesa. Arq. Bras. Med. Vet. Zootec., v.56, p.522-528, 2004.

HENDERSON, C.R. Estimation of variance and covariance components. Biometrics, v.9, p.226252, 1953.

HENDERSON, C.R.; QUAAS, R.L. Multiple trait evaluation using relative's records. J. Dairy Sci., v.43, p.1188, 1976.

HIETANEN, H.; OJALA, M. Factors affecting body weight and its association with milk production traits in Finnish Ayrshire and Friesian cows. Acta Agr. Scand., v.45, p.17-25, 1995.

KLASSEN, D.J.; MONARDES, H.G.; JAIRATH, L. et al. Genetics correlations between life production and linearized type in Canadian Holsteins. J. Dairy Sci., v.75, p.22722282, 1992.

LAWSTUEN, D.A.; HANSEN, L.B.; JOHNSON, L. P. Inheritance and relationships of linear type traits for age groups of Holsteins. J. Dairy Sci., v.70, p.1027-1035, 1987.

MCMANUS, C.; SAUERESSIG, M.G. Estudo das características lineares de tipo em gado Holandês em confinamento total no DF: correlações e análise de componentes principais. In: REUNIÃO ANUAL DA SOCIEDADE BRASILEIRA DE ZOOTECNIA, 34., 1997, Juiz de Fora. Anais... Juiz de Fora: SBZ, 1997. p.19-21.
MEYER, K.; BROTHERSTONE, S.; HILL W.G. et al. Inheritance of linear traits in dairy cattle and correlations with milk production. Anim. Prod., v.44, p.1-10, 1987.

MISZTAL, I.; LAWLOR, T.J.; SHORT, T.H. et al. Multiple-trait estimation of variance components of yield and type traits using an animal model. J. Dairy Sci., v.75, p.544-551, 1992.

MRODE, R.A.; SWANSON, G.J.T. Genetic and phenotypic relationships between conformation and production traits in Ayrshire heifers. Anim. Prod., v.58, p.335-338, 1994.

NORMAN, H.D.; VAN VLECK, L.D. Type appraisal: I. Effects of age and stage of lactation on type ratings. J. Dairy Sci., v.55, p.1706-1716, 1972.

PERSAUD, P.; SIMM, G.; HILL, W. G. Genetic and phenotypic parameters for yield, food intake and efficiency of dairy cows fed ad libitum. Anim. Prod., v.52, p.735-444, 1991.

SCHAEFFER, L.R. Estimates of variance and covariance components for Holstein type traits. Can. J. Anim. Sci., v.63, p.763-771, 1983.

SHORT, T.H.; LAWLOR, JR, T.J.; LEE, K.L. Genetic parameters for three experimental linear type traits. J. Dairy Sci., v.74, p.2020-2025, 1991.

SHORT, T.H.; LAWLOR, T.J. Genetic parameters of conformation traits, milk yield, and herd life in Holsteins. J. Dairy Sci., v.75, p.1987-1998, 1992.

YOUNG, C.W.; LEGATES, J.E.; LECCE, J.C. Genetic and phenotypic relationships between clinical mastitis, laboratory criteria, and udder height. J. Dairy Sci., v.43, p.54-62, 1960.

ZWAAG, H.V.D. Linear type traits in The Netherlands. In: MINAS LEITE - QUALIDADE DO LEITE E PRODUTIVIDADE DE REBANHOS LEITEIROS, 1., 1999, Juiz de Fora. Anais... Juiz de Fora: Embrapa Gado de Leite, 1999. p.63-66. 